\title{
Design, Synthesis, Molecular Modelling, and Biological Evaluation of Oleanolic Acid-Arylidene Derivatives as Potential Anti-Inflammatory Agents
}

This article was published in the following Dove Press journal: Drug Design, Development and Therapy

\author{
Reyaz Hassan Mir' \\ Goutami Godavari ${ }^{2}$ \\ Nasir Ali Siddiqui ${ }^{3}$ \\ Bilal Ahmad (D) ${ }^{4}$ \\ Ramzi A Mothana ${(\mathbb{D})^{3}}^{3}$ \\ Riaz Ullah ${ }^{3}$ \\ Omer M Almarfadi ${ }^{3}$ \\ Sanjay M Jachak ${ }^{2}$ \\ Mubashir Hussain Masoodi (iD) \\ 'Pharmaceutical Chemistry Division, \\ Department of Pharmaceutical Sciences, \\ University of Kashmir, Srinagar, India; \\ ${ }^{2}$ Department of Natural Products, \\ National Institute of Pharmaceutical \\ Education and Research (NIPER), \\ Sahibzada Ajit Singh Nagar, India; \\ ${ }^{3}$ Department of Pharmacognosy, College \\ of Pharmacy, King Saud University, \\ Riyadh, Kingdom of Saudi Arabia; \\ ${ }^{4}$ Department of Molecular Science and \\ Technology, Ajou University, Suwon, \\ South Korea
}

Correspondence: Mubashir Hussain Masoodi

Pharmaceutical Chemistry Division, Department of Pharmaceutical Sciences, University of Kashmir, Hazratbal, Srinagar, Kashmir, 190006, India

Tel +919419076525

Email mubashir@kashmiruniversity.ac.in
Introduction: Oleanolic acid, a pentacyclic triterpenic acid, is widely distributed in medicinal plants and is the most commonly studied triterpene for various biological activities, including anti-allergic, anti-cancer, and anti-inflammatory.

Methods: The present study was carried out to synthesize arylidene derivatives of oleanolic acid at the C-2 position by Claisen Schmidt condensation to develop more effective antiinflammatory agents. The derivatives were screened for anti-inflammatory activity by scrutinizing NO production inhibition in RAW 264.7 cells induced by LPS and their cytotoxicity. The potential candidates were further screened for inhibition of LPS-induced interleukin (IL6) and tumour necrosis factor-alpha (TNF- $\alpha$ ) production in RAW 264.7 cells.

Results: The results of in vitro studies revealed that derivatives $3 \mathrm{~d}, 3 \mathrm{e}, 3 \mathrm{~L}$, and $3 \mathrm{o}$ are comparable to that of the oleanolic acid on the inhibition of TNF- $\alpha$ and IL- 6 release. However, derivative $3 \mathrm{~L}$ was identified as the most potent inhibitor of IL-6 (77.2\%) and TNF- $\alpha(75.4 \%)$ when compared to parent compound, and compounds $3 \mathrm{a}(77.18 \%)$, 3d $(71.5 \%)$, and $3 \mathrm{e}(68.8 \%)$ showed potent inhibition of NO than oleanolic acid $(65.22 \%)$ at $10 \mu \mathrm{M}$. Besides, from docking score and Cyscore analysis analogs (3e, 3L, 3n) showed greater affinity towards TNF- $\alpha$ and IL- $1 \beta$ than dexamethasone.

Conclusion: Herein, we report a series of 15 new arylidene derivatives of oleanolic acid by Claisen Schmidt condensation reaction. All the compounds synthesized were screened for their anti-inflammatory activity against NO, TNF- $\alpha$ and IL-6. From the data, it was evident that most of the compounds exhibited better anti-inflammatory activity.

Keywords: LPS, natural products, IL-1 $\beta$, IL-6, inflammation, RAW 264.7 cells

\section{Introduction}

Inflammation is a combination of highly regulated sequences of events provoked by a variety of stimuli which include microbial, allergic, metabolic, autoimmune, constitutive and physical factors. The series of events are distinguished by five classical inflammatory signs, including redness, pain, swelling, and heat as described by Celsius. Virchow, later on, added the fifth sign loss of function during the 19th century. The response to inflammatory stimulus includes a vascular response (dilation and increased permeability) and a cellular response (leukocytes migration, cellular activation), the whole process is regulated by inflammatory mediators. First, there is an increase in the pro-inflammatory mediators and then of the anti-inflammatory mediators. Depending on the etiological factor and the balance between the inflammatory mediators will be the progression towards 
healing or injury. ${ }^{1-8}$ Moreover, inflammation is the body's primary response to different harmful stimuli involving the innate and adaptive immune system. However, this physiological side of inflammation relies on the presence of endogenous suppressors of pro-inflammatory signalling pathways. 9

Regardless of the fact that drug design and discovery has a high reliance on synthetic chemistry, contribution of natural products cannot be ignored as they played a prominent role in the discovery of various leads for drug development to treat various human diseases. Moreover, WHO list of essential drugs consists of 252 drugs, of which $11 \%$ are of plant origin. ${ }^{10-22}$ Proper screening of biologically active natural products results in the identification of various bioactive molecules as well as drugs. ${ }^{23-30}$ One such example is the discovery of the first analgesic, anti-inflammatory drug aspirin from the bark of willow tree by Greeks and Romans since 400 BC. In 1989 aspirin (acetylsalicylic acid) was approved as the first drug for the treatment of rheumatic disease. ${ }^{31}$ Triterpenoids represent a group of $\mathrm{C}_{30}$ compounds which are biosynthetically derived from the cyclization of squalene. ${ }^{32}$ Due to the ubiquitous nature and diverse biological activities of triterpenoids, they have been the target of interest worldwide for both chemists as well as biologists. Triterpenoids like ursolic acid, oleanolic acid, betulinic acid, and moronic acid (Figure 1) have been reported to exhibit anti-inflammatory, anti-cancer, anti-allergic, and hepatoprotective properties. ${ }^{12,33-42}$ The presence of the carboxyl group at $\mathrm{C}-17$ position, hydroxyl group at the $\mathrm{C}-3$ position and double bond at $\mathrm{C}-12$ position in pentacyclic triterpenoids make these natural scaffolds susceptible to a variety of chemical transformations. ${ }^{43}$ Oleanolic acid, a pentacyclic triterpenic acid, is widely distributed in medicinal plants and food and is the most commonly studied triterpene for various biological activities. ${ }^{37,38,44}$ On oleanolic acid, substantial structural modifications have been executed to uncover more potent antiinflammatory derivatives. ${ }^{4-47}$

The present study was carried out to synthesize arylidene derivatives of oleanolic acid at the $\mathrm{C}-2$ position to develop more effective anti-inflammatory agents. To

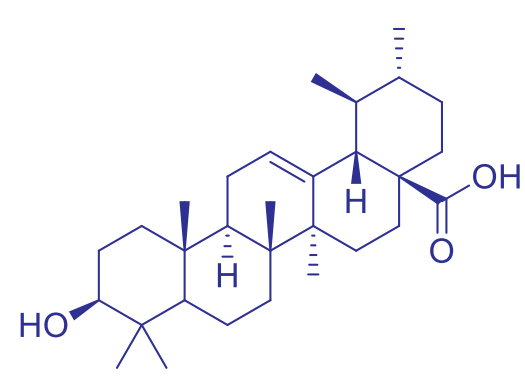

Ursolic acid

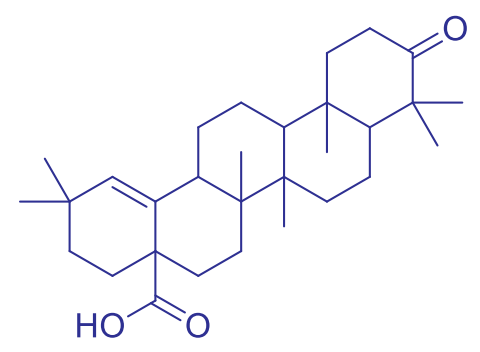

Moronic acid

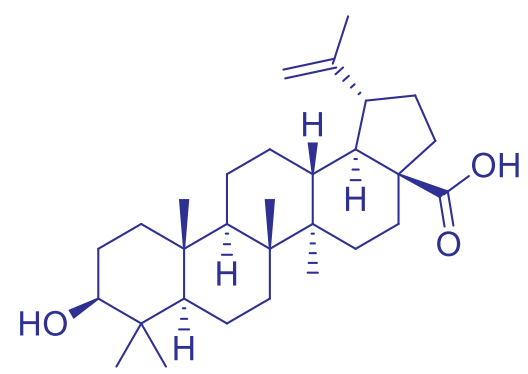

Betulinic acid

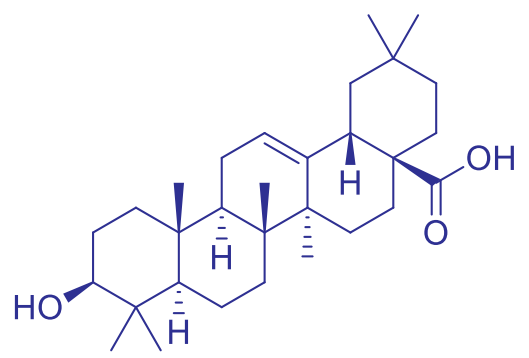

Oleanolic acid

Figure I Some triterpenes with potential biological activity as an anti-inflammatory agent. 
synthesize its various derivatives, oleanolic acid was isolated from Plectranthus rugosus by column chromatography of ethyl acetate fraction followed by recrystallization from methanol. Nmr and mass spectrometry confirmed the structures. The derivatives were screened for antiinflammatory activity by scrutinizing the NO production inhibition in RAW 264.7 cells induced by LPS and their cytotoxicity. The potential candidates were further screened for inhibition of LPS-induced interleukin (IL-6) and tumour necrosis factor-alpha (TNF- $\alpha$ ) production in RAW 264.7 cells.

\section{Experimental Section Materials and Methods}

All the chemicals, as well as reagents, are of high purity. Dulbecco's eagle medium (DMEM) and phosphate buffer saline (PBS) were purchased from Sigma, UK. Elisa kits, IL-6, Human TNF- $\alpha$, were purchased from Invitrogen (USA). From Gibco USA, fetal bovine serum was obtained and lipopolysaccharide (LPS) E. Coli from Calbiochem (USA). Griess reagent was purchased from Promega (USA) and MTT from Calbiochem (San Diego CA). Besides, other chemicals that were used were of research-grade. From the central drug house $(\mathrm{CDH})$ Jammu, ferric chloride, glacial acetic acid, chloroform was purchased. Ascorbic acid, ethanol, methanol, picric acid, calcium chloride, sodium chloride, 2,2 diphenyl picryl hyradyzl (DPPH), sucrose, trichloroacetic acid were procured from Merck. From Qualigens, potassium dihydrogen phosphate, hydrogen peroxide and hydrochloric acid were purchased. Ferric nitrate, ethylene diamine tetraacetate, potassium chloride, sodium hydroxide, dimethyl sulfoxide, sodium dihydrogen monophosphate was purchased from HiMedia. From sisco research laboratories (SRL) butylated hydroxytoluene was bought and from Rankem ethyl acetate and hexane was purchased.

\section{Chemistry}

In the present study, oleanolic acid (1) was isolated from leaf extract of Plectranthus rugosus by column chromatography in 60:40 hexane:ethyl-acetate followed by recrystallization from methanol. The solvents were distilled before using in the reaction. All the reactions were monitored by using $0.2 \mathrm{~mm}$-thick, aluminum-backed TLC plates and were visualized under UV light at $254 \mathrm{~nm}$. The structure was confirmed by nmr and mass spectrometry to give pure white amorphous powder having percentage purity more than $95 \% \mathrm{mp}: 281-283^{\circ} \mathrm{C}[\alpha] \mathrm{D}^{21}$ $+81.3^{\circ}\left(\mathrm{CHCl}_{3} ; \mathrm{c}=0.6\right) .{ }^{1} \mathrm{H}$ NMR $\left(400 \mathrm{MHz}, \mathrm{CDCl}_{3}\right): \delta$ $5.27(1 \mathrm{H}, \mathrm{s}, \mathrm{H}-12), 3.21(\mathrm{IH}, \mathrm{d}, \mathrm{J}=7.2, \mathrm{H}-3), 2.83(1 \mathrm{H}, \mathrm{d}$, $\mathrm{J}=11.3, \mathrm{H}-12), 1.97$ (2H, m, H-2), 0.91 (3H, s, H-23), 0.72 $\left(3 \mathrm{H}, \mathrm{s}, \mathrm{CH}_{3}-24\right), 0.76\left(3 \mathrm{H}, \mathrm{s}, \mathrm{CH}_{3}-25\right), 0.88\left(3 \mathrm{H}, \mathrm{s}, \mathrm{CH}_{3}\right.$ -26), $1.14\left(3 \mathrm{H}, \mathrm{s}, \mathrm{CH}_{3}-27\right), 0.92\left(3 \mathrm{H}, \mathrm{s}, \mathrm{CH}_{3}-29\right), 0.96(3 \mathrm{H}$, $\left.\mathrm{s}, \mathrm{CH}_{3}-30\right) \cdot{ }^{13} \mathrm{C} \mathrm{NMR}\left(100 \mathrm{MHz}, \mathrm{CDCl}_{3}\right): \delta 183.22$ (C-28), 143.62 (C-13), 122.62 (C-12), 79.12 (C-3), 55.24 (C-5), 47.68 (C-9), 46.55 (C-17), 45.7 (C-19), 41.65 (C-14), 40.96 (C-18), 39.12 (C-8), 38.8 (C-1), 37.12 (C-10), 33.86 (C-21), 33.09 (C-29), 32.55 (C-22), 32.59 (C-7), 30.66 (C-20), 28.77 (C-23), 27.9 (C-15), 27.8 (C-2), 25.49 (C-27), 23.89 (C-30), 23.44 (C-16), 22.98 (C-11), 18.33 (C-6), 17.38 (C-26), 15.55 (C-25), 15.38 (C-24). ESI-MS m/z $456[\mathrm{M}+\mathrm{H}]^{+}$

\section{Synthesis of Arylidene-Oleanolic Acid Derivatives}

The protocol for synthesis of oleanolic acid-arylidene derivatives 3 from the parent compound oleanolic acid 1 involves two steps which includes oxidation with pyridinium chlorochromate (PCC) reagent followed by Claisen Schmidt condensation (Scheme 1). So far, many synthetic strategies for the preparation of oleanolic acid analogs have been reported. ${ }^{48-52}$ In a typical procedure, to a solution of compound 1 (400 $\mathrm{mg}, 0.87 \mathrm{mmol})$ in dichloromethane (DCM) at room temperature (rt) few drops of PCC reagent were added. The reaction was monitored by TLC till its completion in $4 \mathrm{~h}$. The reaction mixture was filtered to remove insoluble residue after quenching with cold water. The filtrate was extracted with ethyl-acetate and the organic layer was finally dried over sodium sulphate, concentrated in vaccum and purified by column chromatography to finally give compound 2 (365 $\mathrm{mg} \mathrm{91 \%} \mathrm{yield)} \mathrm{as} \mathrm{pure} \mathrm{white} \mathrm{amorphous}$ solid; mp $179-183^{\circ} \mathrm{C} ;{ }^{1} \mathrm{H}$ NMR $\left(400 \mathrm{MHz}, \mathrm{CDCl}_{3}\right): \delta$ $5.27(1 \mathrm{H}, \mathrm{s}, \mathrm{H}-12), 3.22(1 \mathrm{H}, \mathrm{d}, J=12.0 \mathrm{~Hz}, \mathrm{H}-18), 1.90$ $(2 \mathrm{H}, \mathrm{m}), 1.82(4 \mathrm{H}, \mathrm{m}), 1.55(12 \mathrm{H}, \mathrm{m}), 1.33(4 \mathrm{H}, \mathrm{m}) 1.05$ (3H, s, CH3-23), 0.94 (3H, s, CH3-24), 0.86 (3H, s, CH325), $0.84(3 \mathrm{H}, \mathrm{s}, \mathrm{CH} 3-26), 0.82(3 \mathrm{H}, \mathrm{s}, \mathrm{CH} 3-27), 0.79(3 \mathrm{H}$, s,CH3-29), 0.65 (3H, s,CH3-30); ${ }^{13} \mathrm{C}$ NMR (100 MHz, $\left.\mathrm{CdCl}_{3}\right): \delta \quad 215.7,181.25,151.29,123.32,53.07,52.19$, 51.18, 50.07, 49.09, 47.29, 46.48, 41.34, 40.22, 39.55, $39.29,39.18,39.15,39.12,39.08,38.39,38.24,38.18$, $36.33,28.19,23.37,21.19,17.44,17.78,16.59,15.24$ ESI-MS m/z $455[\mathrm{M}+\mathrm{H}]^{+}$ 


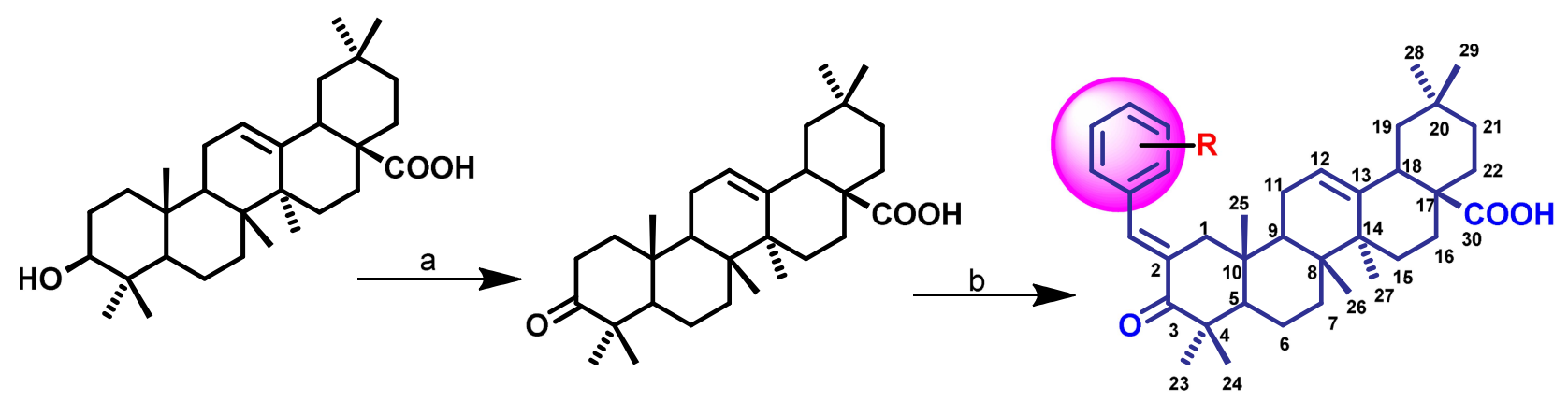

1

2

$3 a-30$

Scheme I Reagents and conditions (a) PCC, DCMrt, 90\% (b) Ar-CHO, LiOH, Ethanol, rt, 80\%.

To the solution of compound 2 ( $365 \mathrm{mg}, 0.8 \mathrm{mmol}$ ), in ethanol at room temperature lithium hydroxide $\mathrm{LiOH}(1.2$ Eq) was added. To this mixture, various aromatic aldehydes were added (Table 1) by Claisen Schmidt condensation reaction (Scheme 1). The crude mixture was extracted with ethyl-acetate, and the organic layer was dried over sodium sulphate, concentrated in vacuum and purified by column chromatography to give pure 3a-3o in $80-90 \%$ yield

\section{3-Oxo-2-[4-Fluorobenzylidenyl]-Olean-12-En-28-Oic Acid (3a)}

Yield 92\%; mp 159-161; ${ }^{1} \mathrm{H}$ NMR (400 MHz, $\mathrm{CDCl}_{3}$ ): $\delta$ $7.57(1 \mathrm{H}, \mathrm{s}), 7.39(2 \mathrm{H}, \mathrm{m}), 7.37(2 \mathrm{H}, \mathrm{m}), 5.28(1 \mathrm{H}, \mathrm{s}), 2.35$ $(2 \mathrm{H}, \mathrm{m}), 2.27(1 \mathrm{H}, \mathrm{d}, \mathrm{j}=8.0 \mathrm{~Hz}), 1.93(4 \mathrm{H}, \mathrm{m}), 1.78(3 \mathrm{H}$, s, CH3-23), 1.63 (3H, s, CH3-24), 1.45 (3H, s, CH3-25), 1.39 (3H, s, CH3-26). 1.09 (3H, s, CH3-27), 0.85 (3H, s, CH3-29), 0.48 (3H, s, CH3-30). ${ }^{13} \mathrm{C}$ NMR (100 MHz, $\left.\mathrm{CDCl}_{3}\right): \delta 207.56,181.08,142.24,138.66,135.31$, $132.19,132.19,130.55,129.22,129.22,123.87,123.13$, $55.22,51.49,48.89,47.14,46.29,42.33,40.87,39.66$, $39.25,38.98,37.46,34.22,33.68,30.45,29.18,26.34$, 24.45, 23.97, 23.77, 20.21, 19.34, 18.92, 18.23, 16.45 . ESI-MS m/z $561[\mathrm{M}+\mathrm{H}]^{+}$

\section{3-Oxo-2-[3-Bromobenzylidenyl]-Olean- I 2-En-28-}

\section{Oic Acid (3b)}

Yield 93\%; mp 148-151; ${ }^{1} \mathrm{HNMR}\left(400 \mathrm{MHz}, \mathrm{CDCl}_{3}\right)$ : $\delta$ $7.48(1 \mathrm{H}, \mathrm{s}), 7.36(2 \mathrm{H}, \mathrm{m}), 7.22(2 \mathrm{H}, \mathrm{m}), 5.32(1 \mathrm{H}, \mathrm{s}), 2.45$ $(1 \mathrm{H}, \mathrm{m}), 2.42(2 \mathrm{H}, \mathrm{m}), 2.23(1 \mathrm{H}, j=8.0 \mathrm{~Hz}), 1.92(4 \mathrm{H}, \mathrm{m})$, $1.72(3 \mathrm{H}, \mathrm{s}, \mathrm{CH} 3-23), 1.62(3 \mathrm{H}, \mathrm{s}, \mathrm{CH} 3-24), 1.42(3 \mathrm{H}, \mathrm{s}$, CH3-25), 1.33 (3H, s, CH3-26). 1.08 (3H, s, CH3-27), 0.80 (3H, s, CH3-29), 0.44 (3H, s, CH3-30). ${ }^{13} \mathrm{C}$ NMR $\left(100 \mathrm{MHz}, \mathrm{CDCl}_{3}\right): \delta 207.91,183.84,143.09,138.43$,
$137.97,136.18,135.34,134.67,130.29,129.96,128.66$, $128.19,127.97,127.18,125.55,125.08,64.78,53.33$, $52.88,48.28,45.55,43.94,42.33,39.66,39.34,39.08$,

Table I Oleanolic Acid-Arylidene Derivatives Varying at the Aromatic Ring at the C-2 Position

\begin{tabular}{|c|c|c|c|}
\hline Compound & $\mathbf{R}$ & $\begin{array}{l}\text { Time of Reaction } \\
\text { (h) }\end{array}$ & $\begin{array}{l}\text { Yield } \\
\text { (\%Age) }\end{array}$ \\
\hline $3 \mathbf{a}$ & & 03 & $92 \%$ \\
\hline 3b & & 02 & $93 \%$ \\
\hline $3 c$ & & 04 & $83 \%$ \\
\hline $3 d$ & & 2.5 & $88 \%$ \\
\hline $3 \mathbf{e}$ & & 04 & $92 \%$ \\
\hline $3 \mathrm{f}$ & & 03 & $81 \%$ \\
\hline $3 \mathrm{~g}$ & & 03 & $87 \%$ \\
\hline
\end{tabular}

(Continued) 
Table I (Continued).

\begin{tabular}{|c|c|c|c|}
\hline Compound & $\mathbf{R}$ & $\begin{array}{l}\text { Time of Reaction } \\
\text { (h) }\end{array}$ & $\begin{array}{l}\text { Yield } \\
\text { (\%Age) }\end{array}$ \\
\hline $3 \mathrm{~h}$ & & 02 & $80 \%$ \\
\hline $3 \mathbf{i}$ & & 05 & $91 \%$ \\
\hline $3 \mathbf{j}$ & & 03 & $86 \%$ \\
\hline $3 \mathbf{k}$ & & 03 & $92 \%$ \\
\hline 3L & & 05 & $87 \%$ \\
\hline $3 m$ & & 04 & $88 \%$ \\
\hline $3 n$ & & 02 & $81 \%$ \\
\hline 30 & & 03 & $83 \%$ \\
\hline
\end{tabular}

$36.87,36.64,32.28,30.85,29.86,29.67,28.17,24.22$, 23.77, 22.94, 21.32, 20.44,17.27. ESI-MS $\mathrm{m} / \mathrm{z} 622$ $[\mathrm{M}+\mathrm{H}]^{+}$

\section{3-Oxo-2-[3-Chlorobenzylidenyl]-Olean-I2-En-28- Oic Acid (3c)}

Yield 83\%; mp 178-181; ${ }^{1} \mathrm{H}$ NMR (400 $\left.\mathrm{MHz}, \mathrm{CDCl}_{3}\right)$ : $\delta$ $7.50(1 \mathrm{H}, \mathrm{s}), 7.31(2 \mathrm{H}, \mathrm{m}), 7.25(2 \mathrm{H}, \mathrm{m}), 5.31(1 \mathrm{H}, \mathrm{s}), 2.43$ $(1 \mathrm{H}, \mathrm{m}), 2.41(2 \mathrm{H}, \mathrm{m}), 2.27(1 \mathrm{H}, \mathrm{d}, \mathrm{j}=8.0 \mathrm{~Hz}), 1.96$ $(4 \mathrm{H}, \mathrm{m}), 1.76(3 \mathrm{H}, \mathrm{s}, \mathrm{CH} 3-23), 1.61(3 \mathrm{H}, \mathrm{s}, \mathrm{CH} 3-24)$, $1.46(3 \mathrm{H}, \mathrm{s}, \mathrm{CH} 3-25), 1.38(3 \mathrm{H}, \mathrm{s}, \mathrm{CH} 3-26) .1 .04(3 \mathrm{H}, \mathrm{s}$, CH3-27), 0.65 (3H, s, CH3-29), 0.58 (3H, s, CH3-30). ${ }^{13} \mathrm{C}$ NMR (100 MHz, $\left.\mathrm{CDCl}_{3}\right): \delta$ 207.94, 183.86, 143.04, $138.35,137.97,136.15,135.34,134.65,130.23,129.98$, $128.63,128.16,127.93,127.93,127.17,125.60,125.05$, $64.71,53.37,52.81,48.27,45.54,43.95,42.39,39.64$, $39.33,39.05,36.87,36.64,32.28,30.86,29.88,29.66$,
28.18, 24.27, 23.77, 22.91, 21.37, 20.48, 17.26. ESI-MS $\mathrm{m} / \mathrm{z} 578[\mathrm{M}+\mathrm{H}]^{+}$

\section{3-Oxo-2-[4-Nitrobenzylidenyl]-Olean- I2-En-28-Oic} Acid (3d)

Yield 88\%; mp 154-156; ${ }^{1} \mathrm{H}$ NMR (400 MHz, $\left.\mathrm{CDCl}_{3}\right)$ : $\delta$ $7.62(1 \mathrm{H}, \mathrm{s}), 7.45(2 \mathrm{H}, \mathrm{m}), 7.31(1 \mathrm{H}, \mathrm{m}), 7.22(1 \mathrm{H}, \mathrm{m})$, $5.31(1 \mathrm{H}, \mathrm{s}), 2.41(2 \mathrm{H}, \mathrm{m}), 2.27(1 \mathrm{H}, \mathrm{d}, j=8.0 \mathrm{~Hz}), 1.94$ (4H, m), 1.79 (3H, s, CH3-23), 1.66 (3H, s, CH3-24), 1.49 (3H, s, CH3-25), 1.29 (3H, s, CH3-26). 1.04 (3H, s, CH327), 0.80 (3H, s, CH3-29), 0.43 (3H, s, CH3-30). ${ }^{13} \mathrm{C}$ NMR $\left(100 \mathrm{MHz}, \mathrm{CDCl}_{3}\right): \delta 207.53,181.04,142.22,137.45$, $134.81,133.44,129.77,129.53,129.42,128.13,126.28$, $123.02,55.23,51.97,48.92,47.19,46.23,42.77,40.75$, $39.66,39.38,38.97,37.55,34.44,33.91,30.22,29.18$, $26.62,24.28,23.91,23.77,20.02,19.44,18.66,18.19$, 16.82. ESI-MS m/z $588[\mathrm{M}+\mathrm{H}]^{+}$

\section{3-Oxo-2-[4-Chlorobenzylidenyl]-Olean- I2-En-28- Oic Acid (3e)}

Yield 92\%; mp 183-185; ${ }^{1} \mathrm{H}$ NMR (400 MHz, $\mathrm{CDCl}_{3}$ ): $\delta$ $7.52(1 \mathrm{H}, \mathrm{s}), 7.33(2 \mathrm{H}, \mathrm{m}), 7.21(1 \mathrm{H}, \mathrm{m}), 7.12(1 \mathrm{H}, \mathrm{m})$, $5.41(1 \mathrm{H}, \mathrm{s}), 2.42(1 \mathrm{H}, \mathrm{m}), 2.41(2 \mathrm{H}, \mathrm{m}), 2.28(1 \mathrm{H}, \mathrm{d}, j=$ $8.0 \mathrm{~Hz}), 1.92$ (4H, m), 1.72 (3H, s, CH3-23), 1.60 (3H, s, CH3-24), 1.48 (3H, s, CH3-25), 1.32 (3H, s, CH3-26). 1.12 (3H, s, CH3-27), 0.88 (3H, s, CH3-29), 0.44 (3H, s, CH3-30). ${ }^{13} \mathrm{C}$ NMR $\left(100 \mathrm{MHz}, \mathrm{CDCl}_{3}\right) \delta 207.52,181.05$, 142.21, 138.66, 135.56, 134.14, 130.52, 129.30, 129.28, 123.09, 55.28, 51.92, 48.94, 47.59, 46.22, 42.77, 40.79, $39.38,39.33,38.97,37.55,34.44,33.92,30.29,29.19$, 26.66, 24.28, 23.92, 23.71, 20.09, 19.66, 18.63, 18.15, 16.89. ESI-MS m/z $578[\mathrm{M}+\mathrm{H}]^{+}$

\section{3-Oxo-2-[2-Methoxybenzylidenyl]-Olean- I 2-En-28- Oic Acid (3f)}

Yield 81\%; mp 171-174; ${ }^{1} \mathrm{H}$ NMR (400 MHz, $\mathrm{CDCl}_{3}$ ): $\delta$ $7.46(1 \mathrm{H}, \mathrm{s}), 7.32(2 \mathrm{H}, \mathrm{m}), 7.12(1 \mathrm{H}, \mathrm{m}), 6.96(1 \mathrm{H}, \mathrm{m}), 5.32$ $(1 \mathrm{H}, \mathrm{s}), 3.83(3 \mathrm{H}, \mathrm{s}), 2.88(1 \mathrm{H}, \mathrm{m}), 2.43(2 \mathrm{H}, \mathrm{m}), 2.28(1 \mathrm{H}$, $\mathrm{d}, j=8.0 \mathrm{~Hz}), 1.98(4 \mathrm{H}, \mathrm{m}), 1.79(3 \mathrm{H}, \mathrm{s}, \mathrm{CH} 3-23), 1.65(3 \mathrm{H}$, s, CH3-24), 1.48 (3H, s, CH3-25), 1.39 (3H, s, CH3-26). 1.09 (3H, s, CH3-27), 0.85 (3H, s, CH3-29), $0.48(3 \mathrm{H}, \mathrm{s}$, CH3-30). ${ }^{13} \mathrm{C}$ NMR (100 MHz, $\mathrm{CDCl}_{3}$ ): $\delta$ 207.91, 183.88, $159.71,138.27,137.44,134.33,129.49,125.88,122.82$, $116.04,114.19,55.49,53.48,52.98,48.29,45.42,44.28$, $42.43,39.59,39.49,39.07,36.98,36.56,32.31,30.77$, $29.88,28.27,24.39,23.77,22.99,21.44,20.55,17.33$, 17.09, 15.66. ESI-MS m/z $573[\mathrm{M}+\mathrm{H}]^{+}$ 


\section{3-Oxo-2-[2-Trifluoromethylbenzylidenyl]-Olean- I2-} En-28-Oic Acid (3g)

Yield 87\%; mp 177-179; ${ }^{1} \mathrm{H}$ NMR (400 MHz, $\left.\mathrm{CDCl}_{3}\right)$ : $\delta$ $7.58(1 \mathrm{H}, \mathrm{s}), 7.52(2 \mathrm{H}, \mathrm{m}), 7.41(1 \mathrm{H}, \mathrm{m}), 7.32(1 \mathrm{H}, \mathrm{m})$, $5.28(1 \mathrm{H}, \mathrm{s}), 2.48(1 \mathrm{H}, \mathrm{m}), 2.46(2 \mathrm{H}, \mathrm{m}), 2.26(1 \mathrm{H}, \mathrm{d}, j=$ $8.0 \mathrm{~Hz}), 1.94(4 \mathrm{H}, \mathrm{m}), 1.75(3 \mathrm{H}, \mathrm{s}, \mathrm{CH} 3-23), 1.62(3 \mathrm{H}, \mathrm{s}$, CH3-24), 1.44 (3H, s, CH3-25), 1.30 (3H, s, CH3-26). 1.19 (3H, s, CH3-27), 0.95 (3H, s, CH3-29), 0.38 (3H, s, CH3-30). ${ }^{13} \mathrm{C}$ NMR $\left(100 \mathrm{MHz}, \mathrm{CDCl}_{3}\right): \delta 207.92,183.84$, $159.77,138.29,137.47,134.36,129.47,125.86,122.85$, $116.09,114.16,55.47,53.44,52.96,48.24,45.44,44.27$, $42.42,39.53,39.45,39.09,36.93,36.52,32.37,30.79$, $29.82,28.24,24.32,23.79,22.91,21.42,20.59,17.32$, 17.07, 15.61 ESI-MS m/z $611[\mathrm{M}+\mathrm{H}]^{+}$

\section{3-Oxo-2-[4-Bromobenzylidenyl]-Olean-I2-En-28- \\ Oic Acid (3h)}

Yield 80\%; mp 163-166; ${ }^{1} \mathrm{H}$ NMR (400 MHz, $\left.\mathrm{CDCl}_{3}\right)$ : $\delta$ $7.54(1 \mathrm{H}, \mathrm{s}), 7.32(2 \mathrm{H}, \mathrm{m}), 7.22(1 \mathrm{H}, \mathrm{m}), 7.14(1 \mathrm{H}, \mathrm{m})$, $5.43(1 \mathrm{H}, \mathrm{s}), 2.45(1 \mathrm{H}, \mathrm{m}), 2.42(2 \mathrm{H}, \mathrm{m}), 2.26(1 \mathrm{H}, \mathrm{d}, j=$ $8.0 \mathrm{~Hz}), 1.94(4 \mathrm{H}, \mathrm{m}), 1.74(3 \mathrm{H}, \mathrm{s}, \mathrm{CH} 3-23), 1.64(3 \mathrm{H}, \mathrm{s}$, CH3-24), 1.42 (3H, s, CH3-25), 1.34 (3H, s, CH3-26). 1.14 (3H, s, CH3-27), 0.82 (3H, s, CH3-29), 0.42 (3H, s, CH3-30) ${ }^{13} \mathrm{C}$ NMR $\left(100 \mathrm{MHz}, \mathrm{CDCl}_{3}\right): \delta 207.51,181.06$, $142.22,138.68,135.57,134.11,130.53,129.35,129.23$, 123.04, 55.23, 51.96, 48.93, 47.54, 46.21, 42.78, 40.73, $39.34,39.34,38.96,37.56,34.47,33.95,30.28,29.11$, $26.63,24.24,23.95,23.78,20.04,19.68,18.67,18.13$, 16.82 ESI-MS m/z $622[\mathrm{M}+\mathrm{H}]^{+}$

\section{3-Oxo-2-[Pyridine-4-Ylmethylene]-Olean-I2-En-28- Oic Acid (3i)}

Yield 91\%; mp 138-141; ${ }^{1} \mathrm{HNMR}\left(400 \mathrm{MHz}, \mathrm{CDCl}_{3}\right): \delta$ $8.57(1 \mathrm{H}, \mathrm{s}), 8.39(\mathrm{H}, \mathrm{m}), 7.48(2 \mathrm{H}, \mathrm{m}), 7.27(1 \mathrm{H}, \mathrm{m}) 5.24$ $(1 \mathrm{H}, \mathrm{s}), 2.39(2 \mathrm{H}, \mathrm{m}), 2.28(1 \mathrm{H}, \mathrm{d}, \mathrm{j}=8.0 \mathrm{~Hz}), 1.98$ $(4 \mathrm{H}, \mathrm{m}), 1.75(3 \mathrm{H}, \mathrm{s}, \mathrm{CH} 3-23), 1.63(3 \mathrm{H}, \mathrm{s}, \mathrm{CH} 3-24)$, $1.45(3 \mathrm{H}, \mathrm{s}, \mathrm{CH} 3-25), 1.39$ (3H, s, CH3-26). $1.09(3 \mathrm{H}, \mathrm{s}$, CH3-27), 0.85 (3H, s, CH3-29), 0.48 (3H, s, CH3-30).${ }^{13} \mathrm{C}$ NMR $\left(100 \mathrm{MHz}, \mathrm{CDCl}_{3}\right): \delta$ 205.56, 180.07, 143.27, 139.67, 136.32, 134.18, 134.18, 131.54, 128.21,128.21, $124.88,124.15,56.21,52.48,49.88,48.18,47.26,43.33$, $41.88,38.65,38.23,36.99,35.43,33.24,32.69,30.46$, $28.19,27.39,25.47,22.99,23.76,21.25,18.36,17.94$, 17.26, 16.43 ESI-MS m/z $544[\mathrm{M}+\mathrm{H}]^{+}$

\section{3-Oxo-2-[Pyridine-3-Ylmethylene]-Olean-I2-En-28-} Oic Acid (3j)

Yield 93\%; mp 148-151; ${ }^{1} \mathrm{H}$ NMR (400 MHz, $\mathrm{CDCl}_{3}$ ): $\delta$ $8.77(1 \mathrm{H}, \mathrm{s}), 8.44(\mathrm{H}, \mathrm{m}), 7.22(2 \mathrm{H}, \mathrm{m}), 7.12(1 \mathrm{H}, \mathrm{m}) 5.33$
$(1 \mathrm{H}, \mathrm{s}), 2.35(2 \mathrm{H}, \mathrm{m}), 2.25(1 \mathrm{H}, \mathrm{d}, \mathrm{j}=8.0 \mathrm{~Hz}), 1.91$ $(4 \mathrm{H}, \mathrm{m}), 1.71(3 \mathrm{H}, \mathrm{s}, \mathrm{CH} 3-23), 1.61$ (3H, s, CH3-24), $1.40(3 \mathrm{H}, \mathrm{s}, \mathrm{CH} 3-25), 1.32$ (3H, s, CH3-26). $1.02(3 \mathrm{H}, \mathrm{s}$, CH3-27), 0.91 (3H, s, CH3-29), 0.41 (3H, s, CH3-30).${ }^{13} \mathrm{C}$ NMR $\left(100 \mathrm{MHz}, \mathrm{CDCl}_{3}\right): \delta 205.46,180.22,143.77$, $139.66,136.43,134.88,134.88,131.87,128.93,128.93$, $124.32,124.32,56.44,52.77,49.84,48.39,47.39,43.28$, $41.89,38.56,38.45,36.89,35.34,33.58,32.79,30.63$, $28.91,27.28,25.78,22.30,23.69,21.59,18.29,17.92$, $17.19,16.54$ ESI-MS m/z $544[\mathrm{M}+\mathrm{H}]^{+}$

\section{3-Oxo-2-[Thiophene-2-YImethylene]-Olean- I 2-En -28-Oic Acid (3k)}

Yield 92\%; mp 184-186; (thiophen-2-ylmethylene)${ }^{1} \mathrm{H}$ NMR (400 MHz, $\left.\mathrm{CDCl}_{3}\right): \delta 8.13(1 \mathrm{H}, \mathrm{s}), 7.22(2 \mathrm{H}, \mathrm{m})$, $7.12(1 \mathrm{H}, \mathrm{m}) 5.23(1 \mathrm{H}, \mathrm{s}), 2.32(2 \mathrm{H}, \mathrm{m}), 2.20(1 \mathrm{H}, \mathrm{d}, \mathrm{j}=$ $8.0 \mathrm{~Hz}), 1.92(4 \mathrm{H}, \mathrm{m}), 1.78(3 \mathrm{H}, \mathrm{s}, \mathrm{CH} 3-23), 1.68(3 \mathrm{H}, \mathrm{s}$, CH3-24), 1.48 (3H, s, CH3-25), 1.37 (3H, s, CH3-26). 1.01 (3H, s, CH3-27), 0.82 (3H, s, CH3-29), 0.49 (3H, s, CH3-30). ${ }^{13} \mathrm{C}$ NMR $\left(100 \mathrm{MHz}, \mathrm{CDCl}_{3}\right): \delta 204.49,180.28$, $148.79,142.68,136.48,134.80,134.83,131.77$, $128.85,128.23,124.56,124.39,54.48,52.57,49.36$, $48.74,47.92,43.93,41.49,38.29,38.19,36.19,35.78$, $33.49,32.49,30.49,28.29,27.48,25.36,22.22,23.82$, $21.57,18.29,17.74, \quad 17.19,16.49$ ESI-MS m/z 549 $[\mathrm{M}+\mathrm{H}]^{+}$

\section{3-Oxo-2-[2,6-Dichlorobenzylidenyl]-Olean- I 2-En -28-Oic Acid (3L)}

Yield 87\%; mp 172-174; ${ }^{1} \mathrm{H}$ NMR (400 MHz, $\left.\mathrm{CDCl}_{3}\right)$ : $\delta$ $7.53(1 \mathrm{H}, \mathrm{s}), 7.31(2 \mathrm{H}, \mathrm{m}), 7.12(1 \mathrm{H}, \mathrm{m}) 5.28(1 \mathrm{H}, \mathrm{s}), 2.36$ $(2 \mathrm{H}, \mathrm{m}), 2.26(1 \mathrm{H}, \mathrm{d}, \mathrm{j}=8.0 \mathrm{~Hz}), 1.97(4 \mathrm{H}, \mathrm{m}), 1.75(3 \mathrm{H}$, s, CH3-23), 1.64 (3H, s, CH3-24), 1.45 (3H, s, CH3-25), 1.32 (3H, s, CH3-26). 1.13 (3H, s, CH3-27), 0.89 (3H, s, CH3-29), 0.40 (3H, s, CH3-30). ${ }^{13} \mathrm{C}$ NMR (100 MHz, $\left.\mathrm{CDCl}_{3}\right): \delta 207.59,181.03,142.29,138.56,135.38$, $132.12,132.10,130.59,129.24,129.24,123.86,123.43$, $55.58,51.42,48.81,47.19,46.25,42.35,40.81,39.64$, $39.21,38.94,37.42,34.27,33.63,30.47,29.16,26.32$, $24.48,23.90,23.79,20.22,19.37,18.97,18.21,16.48$ ESIMS m/z $612[\mathrm{M}+\mathrm{H}]^{+}$

\section{3-Oxo-2-[Naphthalene-I-YImethylene]-Olean-I2-En -28-Oic Acid (3m)}

Yield 88\%; mp 185-187; ${ }^{1} \mathrm{H}$ NMR (400 MHz, $\left.\mathrm{CDCl}_{3}\right)$ : $\delta$ $8.17(2 \mathrm{H}, \mathrm{m}), 7.59(2 \mathrm{H}, \mathrm{m}), 7.47(2 \mathrm{H}, \mathrm{m}), 7.29(1 \mathrm{H}, \mathrm{m})$, $7.13(1 \mathrm{H}, \mathrm{m}) 5.24(1 \mathrm{H}, \mathrm{s}), 2.31(2 \mathrm{H}, \mathrm{m}), 2.20(1 \mathrm{H}, \mathrm{d}, \mathrm{j}=$ $8.0 \mathrm{~Hz}), 1.90(4 \mathrm{H}, \mathrm{m}), 1.71(3 \mathrm{H}, \mathrm{s}, \mathrm{CH} 3-23), 1.68(3 \mathrm{H}, \mathrm{s}$, CH3-24), 1.47 (3H, s, CH3-25), 1.32 (3H, s, CH3-26). 
1.19 (3H, s, CH3-27), 0.86 (3H, s, CH3-29), 0.44 (3H, s, $\mathrm{CH} 3-30) .{ }^{13} \mathrm{C}$ NMR $\left(100 \mathrm{MHz}, \mathrm{CDCl}_{3}\right): \delta 207.87,181.11$, $142.29, \quad 138.78, \quad 135.42, \quad 132.17, \quad 132.11,130.46$, $129.31,129.31,123.78,123.33,55.48, \quad 51.41,48.82$, $47.64,46.82,42.59,40.57,39.33,39.28,38.79,37.29$, $34.19,33.85,30.29,29.18,26.59,24.51,23.75,23.59$, 20.29, 19.29, 18.89, 18.69, 16.54 ESI-MS m/z 593 $[\mathrm{M}+\mathrm{H}]^{+}$

3-Oxo-2-[Benzylidenyl]-Olean- I 2-En-28-Oic Acid (3n) Yield 81\%; mp 144-146; ${ }^{1} \mathrm{H}$ NMR (400 MHz, $\left.\mathrm{CDCl}_{3}\right): \delta$ $7.58(2 \mathrm{H}, \mathrm{m}), 7.39$ (2H, m), 7.37 (1H, m), 7.13 (IH, m), $5.26(1 \mathrm{H}, \mathrm{s}), 2.38(2 \mathrm{H}, \mathrm{m}), 2.21(1 \mathrm{H}, \mathrm{d}, \mathrm{j}=8.0 \mathrm{~Hz}), 1.94$ (4H, m), 1.77 (3H, s, CH3-23), 1.67 (3H, s, CH3-24), 1.46 (3H, s, CH3-25), 1.37 (3H, s, CH3-26). 1.09 (3H, s, CH327), 0.89 (3H, s, CH3-29), 0.47 (3H, s, CH3-30). ${ }^{13} \mathrm{C}$ NMR $\left(100 \mathrm{MHz}, \mathrm{CDCl}_{3}\right): \delta 207.51,181.04,142.28,138.64$, $135.39,132.15,132.14,130.59,129.28,129.28,123.89$, $123.18,55.23,51.41,48.85,47.13,46.27,42.39,40.81$, $39.63,39.26,38.91,37.45,34.29,33.64,30.43,29.16$, $26.33,24.48,23.92,23.73,20.28,19.33,18.99,18.28$, 16.43 ESI-MS m/z $543[\mathrm{M}+\mathrm{H}]^{+}$

\section{3-Oxo-2-[3-Nitrobenzylidenyl]-Olean- I2-En-28-Oic Acid (3o)}

Yield 83\%; mp 161-163; ${ }^{1} \mathrm{H}$ NMR (400 $\left.\mathrm{MHz} \mathrm{CDCl}_{3}\right): \delta$ $8.30(1 \mathrm{H}, \mathrm{s}), 8.13(1 \mathrm{H}, \mathrm{m}), 7.91(2 \mathrm{H}, 1), 7.61(1 \mathrm{H}, \mathrm{m})$, $7.25(1 \mathrm{H}, \mathrm{m}), 5.33(1 \mathrm{H}, \mathrm{s}), 2.46(1 \mathrm{H}, \mathrm{m}), 2.40(2 \mathrm{H}, \mathrm{m})$, $2.24(1 \mathrm{H}, \mathrm{d}, \mathrm{j}=8.0 \mathrm{~Hz}), 1.91(4 \mathrm{H}, \mathrm{m}), 1.79(3 \mathrm{H}, \mathrm{s}, \mathrm{CH} 3-23)$, 1.63 (3H, s, CH3-24), 1.42 (3H, s, CH3-25), 1.31 (3H, s, CH3-26). 1.02 (3H, s, CH3-27), 0.81 (3H, s, CH3-29), $0.42(3 \mathrm{H}, \mathrm{s}, \mathrm{CH} 3-30) .{ }^{13} \mathrm{C} \mathrm{NMR}\left(100 \mathrm{MHz}, \mathrm{CDCl}_{3}\right): \delta$ 207.98, 183.85, 143.05, 138.39, 137.97, 136.19, 135.32, $134.61,130.29,129.96,128.66,128.18,127.92,127.95$, $127.13,125.69,125.05,64.79,53.32,52.89,48.22,45.58$, $43.91,42.35,39.65,39.31,39.07,36.82,36.67,32.27$, $30.83,29.85,29.62,28.17,24.27,23.75,22.93,21.38$, 20.42, 17.28 ESI-MS m/z $588[\mathrm{M}+\mathrm{H}]^{+}$

\section{Biological Activity Evaluation of Cell Viability by MTT Assay}

MTT (3-[4,5-dimethylthiazol-2-yl]-2,5 diphenyltetrazolium bromide) assay was used to study the cytotoxicity of all synthetic compounds against cultured RAW 264.7 cells (purchased commercially from ATCC, Manassas, VA, USA). ${ }^{53}$ Camptothecin was taken as a reference standard in this study. In brief RAW 264.7 cells at a density of 16,000 cells/well were seeded into 96 -well plates and were allowed to adhere in a $\mathrm{CO}_{2}$ incubator at $37^{\circ} \mathrm{C}$ for a time period of $24 \mathrm{~h}$. After $24 \mathrm{~h}$ of incubation, the cells were treated with different concentrations of oleanolic derivatives $(0-10 \mu \mathrm{M} / \mathrm{mL})$ for another $24 \mathrm{~h}$. Afterwards, $20 \mu \mathrm{L}$ of MTT $(0.5 \mathrm{mg} / \mathrm{mL}$ in PBS, PH 7.4) was added and kept for incubation for another $4 \mathrm{~h}$ at $37^{\circ} \mathrm{C}$. Finally, the supernatant was removed, and $100 \mu \mathrm{L}$ of dimethyl sulfoxide (DMSO) was added to each well to dissolve the formazan crystals which are formed after the addition of MTT. The absorbance was measured by using the synergy Mx plate reader at $570 \mathrm{~nm}$. The results were expressed as a percentage of cell viability by using LPS-induced group as a control group. Three replicates were carried for each treatment.

\section{NO Assay}

For the evaluation of NO production in RAW 264.7 cells, the concentration of nitrite was measured in the supernatant as an indicator using Griess reaction. In brief, RAW 264.7 macrophage cells ( $2 \times 10^{5}$ cells/well) were treated with different concentrations of synthetic derivatives in the presence or absence of LPS $(1 \mu \mathrm{g} / \mathrm{mL}) 1$ $\mathrm{h}$ before LPS treatment and then kept in incubation for $24 \mathrm{~h}$. Dexamethasone in different concentrations was used as a positive control. After an incubation period, supernatant $(100 \mu \mathrm{L})$ was collected by centrifugation at $1000 \mathrm{rpm}$ and mixed with Griess reagent $(0.1 \%$ N-1-naphthyl ethylenediamine dihydrochloride and 1\% sulphanilamide in 5\% phosphoric acid) and kept for incubation for $10 \mathrm{~min}$ at room temperature in dark. By using the synergy Mx Plate reader, absorbance was measured at $540 \mathrm{~nm}$. With respect to the standard concentration curve of sodium nitrite $\left(\mathrm{NaNo}_{2}\right)$, concentration of nitrite was calculated. ${ }^{54}$

The percentage inhibition of NO was calculated with the following formula

NO inhibition $(\%)=\frac{(\mathrm{NO} 2) \text { control }-(\mathrm{NO} 2) \text { sample })}{(\mathrm{NO} 2) \text { control }} \times 100$

\section{Measurement of Cytokine Production in RAW 264.7 Cells}

The inhibitory effect of all synthetic derivatives on the production of cytokines (TNF- $\alpha$ and IL-6) was determined by enzyme-linked immunosorbent assay (ELISA) kit. In 96-well plate RAW 264.7 cells were seeded at a density of $2 \times 10^{5}$ cells/well and left for incubation for overnight. The cells were then pretreated with synthetic derivatives for $1 \mathrm{hr}$ before stimulation with LPS for $24 \mathrm{~h}$ to induce inflammation. To collect the supernatant, the culture plate was 
then centrifuged at $1500 \mathrm{rpm}$ and assayed according to the protocol of the manufacturer (Invitrogen) to measure the amount of TNF- $\alpha$ and IL- 6 produced in each sample. The whole experiment was carried out in triplicate.

\section{Molecular Docking and Selection of Predicted Interaction Pose}

Docking of all the synthetic derivatives of oleanolic acid on TNF- $\alpha$ (PDB ID: 2AZ5) and IL-1 $\beta$ (PDB ID: 3040) was performed with molecular operating environment (MOE)2019.01 program. ${ }^{55}$ Structure breaks of TNF- $\alpha$ and IL-1 $\beta$ after retrieved from the RCBS Protein database $^{56}$ were fixed using MOE2019.01. Partial charges and hydrogen atoms were added to both proteins after removing the water molecules. By using the optimized potential for liquid simulations (OPLS) force field in MOE2019.01, the protein structures were minimized. The 3D structure of all the synthetic derivatives of oleanolic acid was prepared and minimized using the MMFF94x force field in MOE2019. ${ }^{57}$ After structure preparation of synthetic derivatives of oleanolic acid and proteins, by using induced-fit docking protocol, the ligands were docked on co-crystallized ligand site in TNF- $\alpha$ and site predicted by MOE and meta pocket $2.0^{58}$ in case of IL- $1 \beta$. All the docked solutions were clustered, and representative solutions of the most populated cluster were selected based on score and subjected to manual selection, based on suitability and binding. The highest scoring solution among manually selected groups of experimentally active compounds were considered as the representative pose. The binding affinity of representative docked pose was calculated using Cyscore. ${ }^{59,60}$

\section{Statistical Analysis}

All the results were expressed as (mean \pm of SD). Graphpad prism eight and microsoft excel was used to calculate SD. P-value of less than 0.5 was considered significant. All the experiments were carried out in triplicates.

\section{Results}

\section{Effect on Cell Viability}

To find the safety level of all the five potent analogs (3d, 3e, 3L, 3n, 3o), MTT assay was performed. Raw cells were subjected to different concentrations $(1-100 \mu \mathrm{M})$ of $(\mathbf{3 d}, \mathbf{3 e}$, 3L, 3n, 3o), for a time period of 48 hours. At low concentrations $(1-10 \mu \mathrm{M})$, we observed that cell viability did not get affected and was more than $80 \%$ at $10 \mu \mathrm{M}$ (Figure 2).

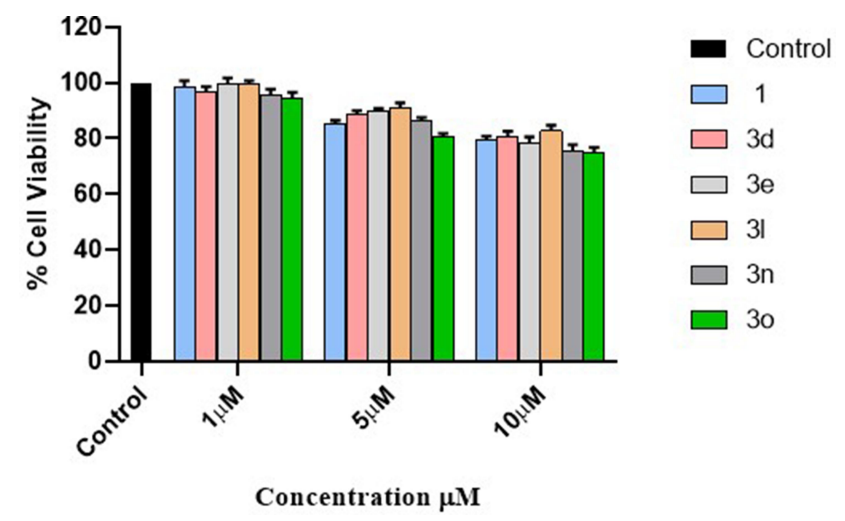

Figure 2 Cytotoxicity of compounds I, 3d, 3e, 3L, 3n and 3o: RAW 264.7 cells were treated up to $10 \mu \mathrm{M}$ concentrations with I, 3d, 3e, $3 \mathrm{~L}, 3 \mathrm{n}$ and 30 for $48 \mathrm{~h}$, and cell viability checked by MTT assay. The data represent mean \pm SD $(n=3)$ of the representative experiment.

Therefore, to investigate the anti-inflammatory of $(\mathbf{3 d}, \mathbf{3 e}$, 3L, 3n, 3o), on RAW 264.7 cells, our data is non-toxic at a concentration of $(1-10 \mu \mathrm{M})$.

\section{Effect of Derivatives on NO Production in LPS Stimulated RAW 264.7 Cells}

Among 15 new derivatives, compounds 3a (77.18\%), 3d (71.5\%) and $3 \mathrm{e}(68.8 \%)$ showed more potent inhibition of NO than oleanolic acid $(65.22 \%)$ at $10 \mu \mathrm{M}$ which is comparable to dexamethasone (85.7\%) and L-NAME (80.5). Analogs 3c, 3k, 3m, 3g, and 3L showed moderate inhibition of NO, respectively. However, analogs $3 \mathrm{~b}, 3 \mathrm{f}, 3 \mathrm{~h}, 3 \mathrm{i}$ and $3 \mathrm{j}$, showed poor inhibitory activity (Table 2 ).

\section{Measurement of Pro-Inflammatory Cytokines (TNF- $\alpha$ and IL-6) in RAW 264.7 Cells}

The synthetic analogs were observed for IL- 6 and TNF- $\alpha$ inhibition. Compounds $3 \mathrm{~d}, 3 \mathrm{e}$, and $3 \mathrm{~L}$ showed maximum IL-6 inhibition of $76.5 \%, 74.9 \%$ and $77.2 \%$ respectively, which is comparable with oleanolic acid (71.6\%) at $10 \mu \mathrm{M}$ concentration. Analogs 3b, 3c, 3h, 3j, 3m, 3n, and $3 \mathrm{o}$ exhibit moderate activity (Table 2), whereas, analogs $3 \mathrm{a}$, $3 \mathrm{f}, 3 \mathrm{~g}, 3 \mathrm{i}, 3 \mathrm{k}$ showed weak IL-6 inhibitory activity. In the case of TNF- $\alpha$, analogs $3 \mathrm{e}, 3 \mathrm{~L}$ and 3o showed maximum inhibitions against TNF- $\alpha$ of $79.3 \%, 75.4 \%$ and $77.1 \%$, which are more potent than oleanolic acid (73.2\%). Others exhibited either moderate or weak activity than oleanolic acid (Table 2). In LPS untreated cells, the levels of TNF- $\alpha$ and IL- 6 were undetectable and served as a control. At $10 \mu \mathrm{M}$ concentration, there were no significant changes in cell viability on the treatment of RAW 
Table 2 The \% Cell Viability and Percentage (\%) Inhibition of Compounds I-I5 Against NO, IL-6, and TNF- $\alpha$ in LPS (I $\mu g / m L)$ Stimulated RAW 246.7 Cells

\begin{tabular}{|c|c|c|c|c|c|c|c|c|}
\hline \multirow{2}{*}{$\frac{\text { S. No. }}{\text { Codes }}$} & \multicolumn{2}{|c|}{$\%$ Cell Viability } & \multicolumn{2}{|c|}{$\%$ Inhibition of NO } & \multicolumn{2}{|c|}{$\%$ Inhibition of TNF-a } & \multicolumn{2}{|c|}{$\%$ Inhibition of IL-6 } \\
\hline & $I \mu M$ & $10 \mu \mathrm{M}$ & $\mathbf{I} \mu \mathrm{M}$ & $10 \mu \mathrm{M}$ & $I \mu M$ & $10 \mu \mathrm{M}$ & $\mathbf{I} \mu \mathrm{M}$ & $10 \mu \mathrm{M}$ \\
\hline I & $98.13 \pm 2.5$ & $78.02 \pm 3.6$ & $15.22 \pm 0.8$ & $65.9 \pm 1.9$ & $25.5 \pm 0.1$ & $73.2 \pm 0.9$ & $26.7 \pm 1.9$ & $71.6 \pm 0.5$ \\
\hline $3 a$ & $90.4 \pm 2.8$ & $74.2 \pm 0.7$ & $17.18 \pm 0.9$ & $77.8 \pm 1.4$ & $3.2 \pm 1.8$ & $20.8 \pm 1.9$ & $7.3 \pm 2.4$ & $18.3 \pm 1.8$ \\
\hline $3 b$ & $91.2 \pm 1.8$ & $71.6 \pm 5.7$ & $11.2 \pm 2.4$ & $14.6 \pm 1.3$ & $4.1 \pm 0.1$ & $19.6 \pm 0.8$ & $13 \pm 1.6$ & $33.3 \pm 2.5$ \\
\hline $3 c$ & $81.1 \pm 2.6$ & $68.2 \pm 2.8$ & $32.6 \pm 3.6$ & $4 I .6 \pm 2.1$ & $9.4 \pm 2.1$ & $17.7 \pm 0.1$ & $11 \pm 1.2$ & $39.5 \pm 2.6$ \\
\hline $3 d$ & $99.2 \pm 4.5$ & $81.7 \pm 1.8$ & $31.5 \pm 6.6$ & $71.5 \pm 2.3$ & $8.7 \pm 2.2$ & $27.7 \pm 0.2$ & $24.4 \pm 1.5$ & $76.5 \pm 0.3$ \\
\hline $3 e$ & $98.22 \pm 2.8$ & $82.9 \pm 3.3$ & $28.8 \pm 4.5$ & $68.8 \pm 3.7$ & $19.1 \pm 0.2$ & $79.3 \pm 0.5$ & $28.5 \pm 1.8$ & $74.9 \pm 2.3$ \\
\hline $3 f$ & $78.6 \pm 1.4$ & $58.3 \pm 2.8$ & $14.3 \pm 18$ & $36.5 \pm 3.9$ & $5.6 \pm 2.8$ & $16.7 \pm 2.1$ & $7.7 \pm 1.4$ & $21.3 \pm 2.7$ \\
\hline $3 g$ & $98.8 \pm 2.5$ & $72.6 \pm 4.5$ & $29.4 \pm 2.3$ & $42.8 \pm 3.7$ & $8.4 \pm 0.9$ & $22.5 \pm 0.5$ & $5.3 \pm 2.4$ & $18.9 \pm 0.7$ \\
\hline $3 \mathrm{~h}$ & $93.6 \pm 3.6$ & $71.1 \pm 2.6$ & $13.4 \pm 2.3$ & $33.6 \pm 3.4$ & $11.3 \pm 1.9$ & $20.1 \pm 1.4$ & $11.2 \pm 1.6$ & $38.6 \pm 0.5$ \\
\hline $3 i$ & $78.4 \pm 1.9$ & $55.9 \pm 4.9$ & $18.2 \pm 2.2$ & $41.4 \pm 0.9$ & $13.6 \pm 0.2$ & $27.5 \pm 0.8$ & $8.9 \pm 0.8$ & $17.6 \pm 1.1$ \\
\hline $3 j$ & $99.3 \pm 4.9$ & $68.4 \pm 3.8$ & $18.3 \pm 3.2$ & $34.6 \pm 0.4$ & $18.8 \pm 0.4$ & $41.7 \pm 0.5$ & $18.6 \pm 0.4$ & $35.9 \pm 1.2$ \\
\hline $3 k$ & $98.1 \pm 2.4$ & $65.6 \pm 3.7$ & $27.2 \pm 2.4$ & $44.2 \pm 3.2$ & $7.2 \pm 2.7$ & $36.7 \pm 2.4$ & $7.8 \pm 1.9$ & $19.6 \pm 2.5$ \\
\hline $3 \mathrm{~L}$ & $99.5 \pm 4.8$ & $82.3 \pm 3.2$ & $21.4 \pm 2.9$ & $42.4 \pm 2.6$ & $25.5 \pm 2.1$ & $75.4 \pm 0.6$ & $32 \pm 1.5$ & $77.2 \pm 2.9$ \\
\hline $3 m$ & $92.2 \pm 5.7$ & $58.9 \pm 2.7$ & $13.12 \pm 2.5$ & $43.2 \pm 1.9$ & $8.9 \pm 2.4$ & $29.9 \pm 1.6$ & $19.8 \pm 1.6$ & $37.6 \pm 2.5$ \\
\hline $3 n$ & $96.6 \pm I .8$ & $84.4 \pm 4.7$ & $14.1 \pm 0.8$ & $42.4 \pm 0.5$ & $8.2 \pm 1.7$ & $35.8 \pm 0.6$ & $29.7 \pm 1.4$ & $55.6 \pm 3.2$ \\
\hline 30 & $97.7 \pm 4.1$ & $83.9 \pm 3.6$ & $21.2 \pm 0.9$ & $46.8 \pm 0.4$ & $19.8 \pm 0.8$ & $77.1 \pm 0.4$ & $8.9 \pm 1.5$ & $51.6 \pm 2.6$ \\
\hline Curcumin & - & - & $43 \pm 0.5$ & $79.5 \pm 0.2$ & $65 \pm 0.1$ & $80 \pm 0.6$ & - & $77.1 \pm 0.9$ \\
\hline Dexa & - & - & $48.7 \pm 2.8$ & $85.7 \pm 1.2$ & $70.9 \pm 2.4$ & $86.2 \pm 3.2$ & - & $82.3 \pm 2.4$ \\
\hline L-NAME & - & - & $44.5 \pm 2.3$ & $80.5 \pm 2.1$ & & & & \\
\hline
\end{tabular}

Note: Compounds in bold font are active.

264.7 cells. Analogs that exhibit more than $50 \%$ inhibition of TNF- $\alpha$ and IL-6 are considered more potent. ${ }^{61,62}$

\section{Molecular Docking Studies}

All the synthetic derivatives were docked on TNF- $\alpha$ and IL-1 $\beta$ (Figure 3A and B). The top-scoring solutions have been analyzed for the Cyscore score, and ADME properties are mentioned in (Table 3). The docking pose shows the interaction of (3d, 3e, 3L, 3n, 3o) and dexamethasone with TNF- $\alpha$ and IL- $\beta$. The binding affinity of analogs and dexamethasone with TNF- $\alpha$ and IL- $1 \beta$ was calculated using Cyscore 2. ${ }^{60,63}$ From docking score and Cyscore analysis, it shows that the analogs $(3 \mathrm{e}, 3 \mathrm{~L}, 3 \mathrm{n})$ are having a greater affinity towards TNF- $\alpha$ and IL- $1 \beta$ than dexamethasone which was also verified from in vitro results.

\section{Discussion}

In the current study, we investigated the various derivatives of oleanolic acid on the production of pro-inflammatory mediators in RAW 264.7 mouse macrophages stimulated by LPS. In RAW 264.7 mouse macrophages, the analogs were evaluated for their potential to reduce lipopolysaccharide (LPS) induced TNF- $\alpha$ and IL-6 production. RAW 264.7 cells upon activation with LPS releases TNF- $\alpha$ and IL-6.
LPS, a component of a gram-negative bacterial cell wall, induces macrophages and monocytes activation which is having a pivotal role in the innate immune response. Stimulation of RAW 264.7 cells with LPS leads to a series of intracellular events which results in the secretion of cytokines as well as other mediators of inflammation that eventually constitute the pro-inflammatory response. RAW 264.7 cells on pretreatment with the analogs (3d, 3e, 3L, 3o) at various concentrations followed by LPS treatment for $24 \mathrm{~h}$ results in the downregulation of pro-inflammatory cytokines (IL-6 and TNF- $\alpha$ ). At $10 \mu \mathrm{g} / \mathrm{mL}$, the analogs showed potent inhibition of cytokines. In LPS untreated cells, the levels of IL- 6 and TNF- $\alpha$ were not detectable and served as a control. MTT assay also showed that up to $10 \mu \mathrm{g} / \mathrm{mL}$ concentration, the analogs $(\mathbf{3 d}, \mathbf{3 e}, \mathbf{3 L}, \mathbf{3 n}, \mathbf{3 0})$ did not affect the viability of RAW 264.7 cells. So the binding of potent analogs ((3d, 3e, 3L, 3n, 3o) results in the downregulation of pro-inflammatory cytokines (TNF- $\alpha$ and IL-6) and upregulated the secretion of anti-inflammatory cytokine IL-10.

Moreover, the analogs (3a, 3d, 3e) also inhibit the production of $\mathrm{NO}$ also, which is also released during the process of inflammation by activated phagocytes or resident cells to activate various macrophages. With reference to standard drug dexamethasone, the inhibitory potential of 
A
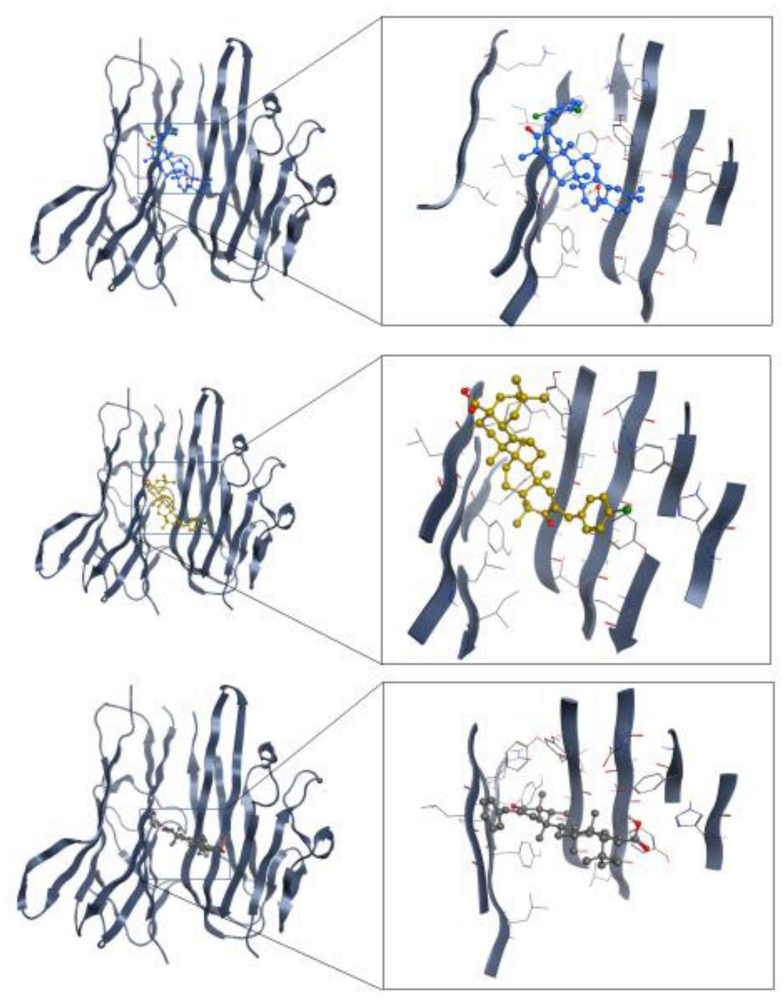

B
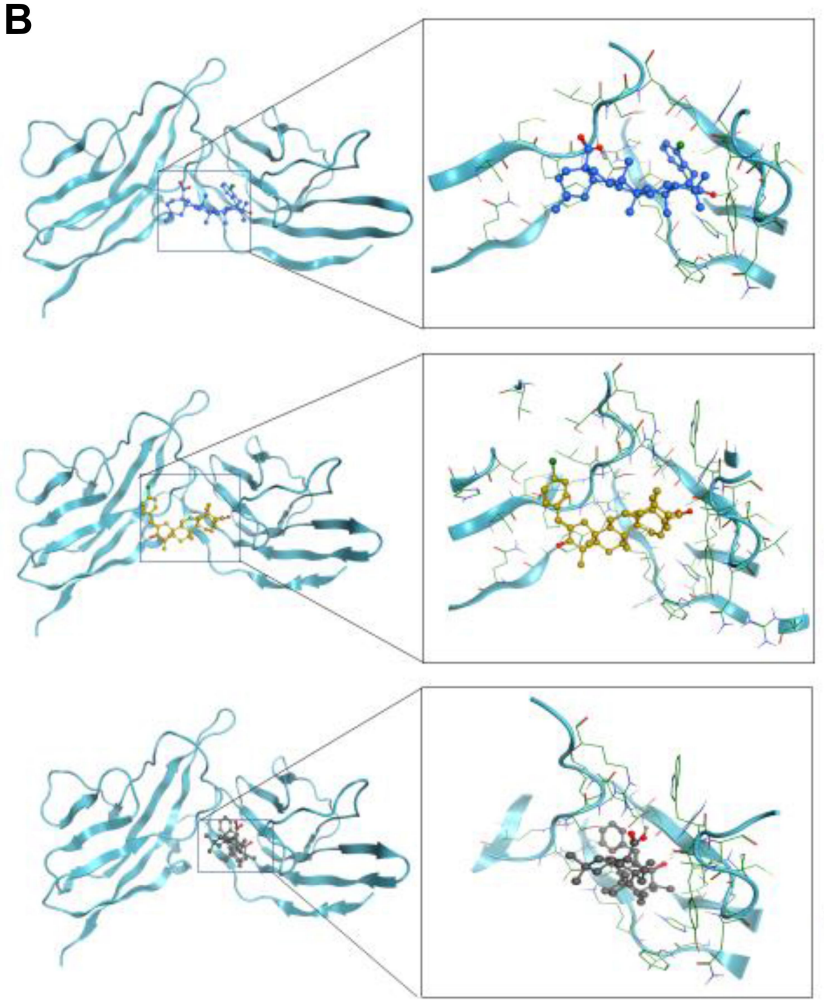

(2) (8) (8)

(:)

$=\underbrace{2}$

(2ii)

2,6 dichloro Benzaldehyde
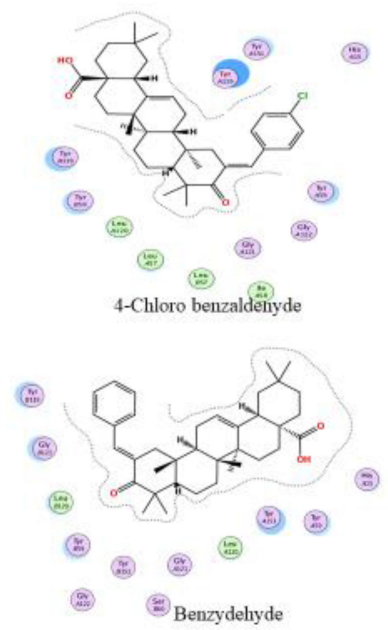

(2) $2 \div$

2,6 dichloro Benzaldehyde

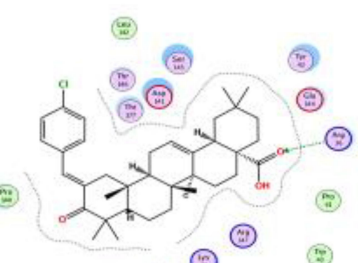

(2) (3) (1)

4-Chloro benzaldehyde

(2) ()

(2) (8)

Figure 3 Interaction of active compounds with TNF- $\alpha$ (A) and IL-I $\beta$ (B). 
Table 3 Docking and Cyscore Score Along with ADME Properties

\begin{tabular}{|c|c|c|c|c|c|c|c|c|c|}
\hline \multirow[t]{2}{*}{ Compound Name } & \multicolumn{2}{|c|}{ Docking Score } & \multicolumn{2}{|c|}{ Cyscore (Binding Affinity) } & \multicolumn{5}{|l|}{ ADME } \\
\hline & TNF- $\alpha$ & IL-I $\beta$ & TNF- $\alpha$ & IL-I $\beta$ & $\log P_{o / w}$ & LogS & $\log k_{p}$ & TPSA & BA Score \\
\hline Dexamethasone & $-5 .|7|$ & -5.934 & -1.974 & -1.319 & 2.15 & 3.56 & 7.32 & 94.83 & 0.55 \\
\hline 4-nitro benzaldehyde (3d) & -7.113 & -7.097 & -2.597 & 3.417 & 6.91 & 9.10 & 3.45 & 100.19 & 0.56 \\
\hline 4-chloro-Benzaldehyde (3e) & -6.653 & -7.253 & -2.334 & 3.257 & 7.39 & 9.10 & 3.26 & 63.60 & 0.56 \\
\hline 2,6 Di-chloro Benzaldehyde (3L) & -7.157 & -7.174 & -1.931 & 3.529 & 8.22 & 10.15 & 2.48 & 54.37 & 0.56 \\
\hline Benzaldehyde (3n) & -6.731 & -6.641 & $-3.05 I$ & 4.004 & 7.47 & 9.00 & 3.06 & 54.37 & 0.56 \\
\hline 3-nitro-Benzaldehyde (3o) & -7.453 & -7.755 & -2.382 & -3.972 & 6.94 & 9.10 & 3.45 & 100.19 & 0.56 \\
\hline
\end{tabular}

Note: Compounds shown in bold font are active.

analogs was studied. Besides, from docking score and Cyscore analysis analogs (3e, 3L, 3n) showed greater affinity towards TNF- $\alpha$ and IL- $1 \beta$ than dexamethasone, which was also verified from our in vitro results.

So we have undertaken a research program directed toward the structural modifications of (1) at C-2 position to fine tune its biological potential as an anti-inflammatory agent. Compound (1) was subjected to oxidation by pyridinium chlorochromate (PCC) reagent at room temperature (rt) that resulted in the formation of (C-3) oxidized derivative (2) in almost quantitative yield. To the solution of compound (2) (365 mg, 0.8 $\mathrm{mmol}$ ), in ethanol at room temperature lithium hydroxide LiOH (1.2 Eq) was added. To this mixture, various aromatic aldehydes were added by Claisen Schmidt condensation reaction. The crude mixture was extracted with ethyl-acetate, and the organic layer was dried over sodium sulphate, concentrated in vacuum and purified by column chromatography to give pure 3a-3o in $80-90 \%$ yield. The products were confirmed by ${ }^{1} \mathrm{H}$ NMR, ${ }^{13} \mathrm{C} \mathrm{NMR}$, and MS analysis. By employing the above reaction conditions, a series of oleanolic acid-arylidene derivatives that vary at substitutions on aromatic ring has been synthesized.

\section{Conclusion}

Herein, we report a series of 15 new arylidene derivatives of oleanolic acid by Claisen Schmidt condensation reaction. All the compounds synthesized were screened for their antiinflammatory activity against NO, TNF- $\alpha$ and IL-6. From the data, it was evident that most of the compounds exhibited better anti-inflammatory activity. Molecular docking studies further confirm the anti-inflammatory activity of the derivatives (3d, 3e, 3L, 3n, 3o). Compounds (3d, 3e, 3L, 3n, and 3o) with o-nitro, o-chloro and p-chloro substitutions were found to be the most promising derivatives. The study also encouraged to study the molecular mechanisms involved, which further demonstrates its anti-inflammatory action at the molecular level.

\section{Acknowledgment}

The authors of this study extend their appreciation to the Research Supporting Project, King Saud University, Saudia Arabia, for supporting this study (RSP-2020/119) and for funding this work. The authors would also like to extend their sincere appreciation to the University of Kashmir, for providing necessary facilities to carry out this work.

\section{Disclosure}

The authors declare no conflicts of interest in this work.

\section{References}

1. Stankov SV. Definition of inflammation, causes of inflammation and possible anti-inflammatory strategies. Open Inflamm J. 2012;5(1):1-9. doi:10.2174/1875041901205010001

2. Tracy RP. The five cardinal signs of inflammation: calor, dolor, rubor, tumor ... and penuria (apologies to Aulus Cornelius Celsus, De medicina, c. AD 25). J Gerontol a Biol Sci Med Sci. 2006;61 (10):1051-1052. doi:10.1093/gerona/61.10.1051

3. Rather L. Disturbance of function (functio laesa): the legendary fifth cardinal sign of inflammation, added by Galen to the four cardinal signs of celsus. Bull N Y Acad Med. 1971;47(3):303.

4. Ferrero-Miliani L, Nielsen O, Andersen P, Girardin S. Chronic inflammation: importance of NOD2 and NALP3 in interleukin-1 $\beta$ generation. Clin Exp Immunol. 2007;147(2):227-235.

5. Hawiger J, Zienkiewicz J. Decoding inflammation, its causes, genomic responses, and emerging countermeasures. Scand J Immunol. 2019;90 (6):e12812. doi:10.1111/sji.12812

6. Taams LS. Inflammation and immune resolution. Clin Exp Immunol. 2018;193(1):1-2. doi:10.1111/cei.13155

7. Koller GM, Schafer C, Kemp SS, et al. Pro-inflammatory mediators, IL (interleukin)-1 $\beta$, TNF (tumor necrosis factor) $\alpha$, and thrombin directly induce capillary tube regression. Arterioscler Thromb Vasc Biol. 2020;40(2):365-377. doi:10.1161/ATVBAHA.119.313536

8. Zanoli L, Briet M, Empana JP, et al. Vascular consequences of inflammation: a position statement from the ESH working group on vascular structure and function and the ARTERY society. J Hypertens. 2020;38 (9):1682-1698. doi:10.1097/HJH.0000000000002508

9. Medzhitov R. Origin and physiological roles of inflammation. Nature. 2008;454(7203):428. doi:10.1038/nature07201 
10. Rates SMK. Plants as source of drugs. Toxicon. 2001;39(5):603-613. doi:10.1016/S0041-0101(00)00154-9

11. Newman DJ, Cragg GM. Natural products as sources of new drugs over the last 25 years. $J$ Nat Prod. 2007;70(3):461-477. doi:10.1021/ np068054v

12. Gosslau A, Li S, Ho CT, Chen KY, Rawson NE. The importance of natural product characterization in studies of their anti-inflammatory activity. Mol Nutr Food Res. 2011;55(1):74-82. doi:10.1002/ mnfr. 201000455

13. Lee KH, Itokawa H, Akiyama T, Morris-Natschke SL. Plant-derived natural products research in drug discovery. Nat Prod Chem Biol. 2012;65:351-388.

14. Spainhour CB. Natural products. Pharm Sci Encycl. 2010;1-62.

15. Cragg GM, Grothaus PG, Newman DJ. Natural products in drug discovery: recent advances. In: Cechinel-Filho V, editor. Plant Bioactives and Drug Discovery: Principles, Practice, and Perspectives. 4th ed. Hoboken: Wiley; 2012:1-42.

16. Strömstedt AA, Felth J, Bohlin L. Bioassays in natural product research-strategies and methods in the search for anti-inflammatory and antimicrobial activity. Phytochem Anal. 2014;25(1):13-28. doi: $10.1002 /$ pca. 2468

17. Lee KH, Itokawa H, Akiyama T, Morris-Natschke SL. Plant-derived natural products research: recent progress in drug discovery. In: Wiley Encyclopedia of Chemical Biology. Hoboken: Wiley; 2007:1-22.

18. Lachance H, Wetzel S, Waldmann H. Role of Natural Products in Drug Discovery. Wiley Online Library; 2010:187-229.

19. Nunes C, Barreto Arantes M, Menezes de Faria Pereira S, et al. Plants as sources of anti-inflammatory agents. Molecules. 2020;25 (16):3726. doi:10.3390/molecules25163726

20. Mir RH, Shah AJ, Mohi-Ud-Din R, et al. Natural Anti-inflammatory compounds as drug candidates in alzheimer's disease. Current Medicinal Chemistry. In press 2020.

21. Hassan R, Masoodi MH. Saussurea lappa: a comprehensive review on its pharmacological activity and phytochemistry. Curr Tradit Med. 2020;6(1):13-23. doi:10.2174/221508380566619062 6144909

22. Mir RH, Masoodi MH. Anti-inflammatory plant polyphenolics and cellular action mechanisms. Curr Bioact Compd. 2020;16 (6):809-817. doi:10.2174/1573407215666190419205317

23. Grabley S, Sattler I. Natural products for lead identification: nature is a valuable resource for providing tools. In: Hillisch A, Hilgenfeld R, editors. Modern Methods of Drug Discovery. Springer; 2003:87-107.

24. Lee K-H. Discovery and development of natural product-derived chemotherapeutic agents based on a medicinal chemistry approach. J Nat Prod. 2010;73(3):500-516. doi:10.1021/np900821e

25. Gautam R, Jachak SM. Recent developments in anti-inflammatory natural products. Med Res Rev. 2009;29(5):767-820.

26. Kobyliak N, Falalyeyeva T, Mykhalchyshyn G, Kyriienko D, Komissarenko I. Effect of alive probiotic on insulin resistance in type 2 diabetes patients: randomized clinical trial. Diabetes Metab Syndr. 2018;12(5):617-624. doi:10.1016/j.dsx.2018.04.015

27. Nance CL. Clinical efficacy trials with natural products and herbal medicines. In: Ramzan I, editor. Phytotherapies: Efficacy, Safety and Regulation. Hoboken: Wiley; 2015:65-88.

28. Freire MO, Van Dyke TE. Natural resolution of inflammation. Periodontology. 2013;63(1):149-164. doi:10.1111/prd.12034

29. Mir RH, Sawhney G, Verma R, et al. Oreganum vulgare: in-vitro assessment of cytotoxicity, molecular docking studies, antioxidant, and evaluation of anti-inflammatory activity in LPS stimulated RAW 264.7 cells. Med Chem. 2020. doi:10.2174/15734064166662009041 10828

30. Bhat MF, Hassan R, Masoodi MH. Nuclear magnetic resonance (NMR) for plant profiling and disease metabolomics-fast tracking plant based drug discovery from northern India. Nucl Magn Reson. 2018;2:1.
31. Vane JR. Inhibition of prostaglandin synthesis as a mechanism of action for aspirin-like drugs. Nat New Biol. 1971;231(25):232-235. doi:10.1038/newbio231232a0

32. Prestwich GD, Abe I, Zheng YF, Robustell BJ, Dang T. Enzymatic cyclization of squalene analogs. Pure Appl Chem. 1999;71 (6):1127-1131. doi:10.1351/pac199971061127

33. Jäger S, Trojan H, Kopp T, Laszczyk M, Scheffler A. Pentacyclic triterpene distribution in various plants - rich sources for a new group of multi-potent plant extracts. Molecules. 2009;14(6):2016-2031. doi:10.3390/molecules 14062016

34. Meng Y-Q, Liu D, Cai -L-L, Chen H, Cao B, Wang Y-Z. The synthesis of ursolic acid derivatives with cytotoxic activity and the investigation of their preliminary mechanism of action. Bioorg Med Chem. 2009;17(2):848-854. doi:10.1016/j.bmc.2008.11.036

35. Kwon T-H, Lee B-M, Chung S-H, Kim D-H, Lee Y-S. Synthesis and NO production inhibitory activities of ursolic acid and oleanolic acid derivatives. Bull Korean Chem Soc. 2009;30(1):119-123.

36. Ikeda Y, Murakami A, Ohigashi H. Ursolic acid: an anti-and proinflammatory triterpenoid. Mol Nutr Food Res. 2008;52(1):26-42. doi:10.1002/mnfr.200700389

37. Liu J. Oleanolic acid and ursolic acid: research perspectives. J Ethnopharmacol. 2005;100(1-2):92-94. doi:10.1016/j.jep.2005.05.024

38. Tian Z, Lin G, Zheng R-X, Huang F, Yang M-S, Xiao P-G. Antihepatoma activity and mechanism of ursolic acid and its derivatives isolated from Aralia decaisneana. World J Gastroenterol. 2006;12 (6):874. doi:10.3748/wjg.v12.i6.874

39. Shyu M-H, Kao T-C, Yen G-C. Oleanolic acid and ursolic acid induce apoptosis in $\mathrm{HuH} 7$ human hepatocellular carcinoma cells through a mitochondrial-dependent pathway and downregulation of XIAP. J Agric Food Chem. 2010;58(10):6110-6118. doi:10.1021/ jf100574j

40. Ma C, Nakamura N, Miyashiro H, Hattori M, Shimotohno K. Inhibitory effects of ursolic acid derivatives from Cynomorium songaricum, and related triterpenes on human immunodeficiency viral protease. Phytother Res. 1998;12(S1):S138-S142.

41. Cos P, Maes L, Vanden Berghe D, Hermans N, Pieters L, Vlietinck A. Plant substances as anti-HIV agents selected according to their putative mechanism of action. $J$ Nat Prod. 2004;67(2):284-293. doi:10.1021/np034016p

42. Yu D, Sakurai Y, Chen C-H, et al. Anti-AIDS agents 69. moronic acid and other triterpene derivatives as novel potent anti-HIV agents. J Med Chem. 2006;49(18):5462-5469. doi:10.1021/jm0601912

43. Pathak A, Singh S, Biabani M, et al. Synthesis of combinatorial libraries based on terpenoid scaffolds. Comb Chem High Throughput Screen. 2002;5(3):241-248. doi:10.2174/1386207024607275

44. Liu J. Pharmacology of oleanolic acid and ursolic acid. JEthnopharmacol. 1995;49(2):57-68. doi:10.1016/0378-8741(95)90032-2

45. Patel NK, Pulipaka S, Dubey SP, Bhutani KK. Pro-inflammatory cytokines and nitric oxide inhibitory constituents from Cassia occidentalis roots. Nat Prod Commun. 2014;9(5):1934578X1400900519.

46. Patel NK, Bhutani KK. Pinostrobin and Cajanus lactone isolated from Cajanus cajan (L.) leaves inhibits TNF- $\alpha$ and IL-1 $\beta$ production: in vitro and in vivo experimentation. Phytomedicine. 2014;21 (7):946-953. doi:10.1016/j.phymed.2014.02.011

47. Bhandari P, Patel NK, Bhutani KK. Synthesis of new heterocyclic lupeol derivatives as nitric oxide and pro-inflammatory cytokine inhibitors. Bioorg Med Chem Lett. 2014;24(15):3596-3599. doi:10.1016/j.bmcl.2014.05.032

48. Ngoc TD, Moons N, Kim Y, et al. Synthesis of triterpenoid triazine derivatives from allobetulone and betulonic acid with biological activities. Bioorg Med Chem. 2014;22(13):3292-3300. doi:10.1016/ j.bmc.2014.04.061

49. Urban M, Vlk M, Dzubak P, Hajduch M, Sarek J. Cytotoxic heterocyclic triterpenoids derived from betulin and betulinic acid. Bioorg Med Chem. 2012;20(11):3666-3674. doi:10.1016/j.bmc.2012.03.066 
50. Haavikko R, Nasereddin A, Sacerdoti-Sierra N, et al. Heterocyclefused lupane triterpenoids inhibit Leishmania donovani amastigotes. MedChemComm. 2014;5(4):445-451. doi:10.1039/C3MD00282A

51. Rashid S, Dar BA, Majeed R, Hamid A, Bhat BA. Synthesis and biological evaluation of ursolic acid-triazolyl derivatives as potential anti-cancer agents. Eur J Med Chem. 2013;66:238-245. doi:10.1016/ j.ejmech.2013.05.029

52. Tang C, Zhu L, Chen Y, et al. Synthesis and biological evaluation of oleanolic acid derivative-chalcone conjugates as $\alpha$-glucosidase inhibitors. RSC Adv. 2014;4(21):10862-10874. doi:10.1039/C3RA464 92J

53. Xu X, Yin P, Wan C, et al. Punicalagin inhibits inflammation in LPSinduced RAW264. 7 macrophages via the suppression of TLR4mediated MAPKs and NF- $\kappa$ B activation. Inflammation. 2014;37 (3):956-965. doi:10.1007/s10753-014-9816-2

54. Joo T, Sowndhararajan K, Hong S, et al. inhibition of nitric oxide production in LPS-stimulated RAW 264.7 cells by stem bark of Ulmus pumila L. Saudi J Biol Sci. 2014;21(5):427-435. doi:10. 1016/j.sjbs.2014.04.003

55. Deeth RJ, Fey N, Williams-Hubbard B. DommiMOE: an implementation of ligand field molecular mechanics in the molecular operating environment. J Comput Chem. 2005;26(2):123-130. doi:10.1002/ jcc. 20137

56. Pappalardo E, Cantone D. Database systems in biology. In: Barolli L, editor. Enterprise Business Modeling, Optimization Techniques, and Flexible Information Systems. IGI Global; 2013:80-96.
57. Heinzerling L, Klein R, Rarey M. Fast force field-based optimization of protein-ligand complexes with graphics processor. J Comput Chem. 2012;33(32):2554-2565. doi:10.1002/jcc.23094

58. Zhang Z, Li Y, Lin B, Schroeder M, Huang B. Identification of cavities on protein surface using multiple computational approaches for drug binding site prediction. Bioinformatics. 2011;27 (15):2083-2088. doi:10.1093/bioinformatics/btr331

59. Cao Y, Dai W, Miao Z. Evaluation of protein-ligand docking by Cyscore. In: Gore M, Jagtap UB, editor. Computational Drug Discovery and Design. Springer; 2018:233-243.

60. Li H, Leung K-S, Wong M-H, Ballester PJ. Substituting random forest for multiple linear regression improves binding affinity prediction of scoring functions: Cyscore as a case study. BMC Bioinform. 2014;15(1):291. doi:10.1186/1471-2105-15-291

61. Ammon H. Modulation of the immune system by Boswellia serrata extracts and boswellic acids. Phytomedicine. 2010;17(11):862-867. doi:10.1016/j.phymed.2010.03.003

62. Lin W-C, Lin J-Y. Five bitter compounds display different anti-inflammatory effects through modulating cytokine secretion using mouse primary splenocytes in vitro. J Agric Food Chem. 2010;59(1):184-192. doi:10.1021/jf103581r

63. Li H, Sze KH, Lu G, Ballester PJ. Machine-learning scoring functions for structure-based drug lead optimization. Wiley Interdiscip Rev Comput Mol Sci. 2020;e1465.
Drug Design, Development and Therapy

\section{Publish your work in this journal}

Drug Design, Development and Therapy is an international, peerreviewed open-access journal that spans the spectrum of drug design and development through to clinical applications. Clinical outcomes, patient safety, and programs for the development and effective, safe, and sustained use of medicines are a feature of the journal, which has also

\section{Dovepress}

been accepted for indexing on PubMed Central. The manuscript management system is completely online and includes a very quick and fair peer-review system, which is all easy to use. Visit http://www. dovepress.com/testimonials.php to read real quotes from published authors. 